\title{
A dramatic difference between the electron-driven dissociation of alcohols and ethers and its relation to Rydberg states
}

\author{
Bogdan C. Ibănescu* and Michael Allan
}

A difference was observed in the reactivity of alcohols and ethers toward free electrons. Whereas the lowest core-excited state of the negative ion - $\mathrm{a}^{2}\left(n, 3 \mathrm{~s}^{2}\right)$ Feshbach resonance- of the alcohols readily dissociates by losing a hydrogen atom, ethers show no observable signal from this resonance. This difference in reactivity has a parallel in the anomalous shapes and energies of the parent states of the Feshbach resonances, the ${ }^{1}(n, 3 \mathrm{~s})$ Rydberg states of the neutral alcohols. We explained this anomaly using potential surfaces of the alcohols and ethers calculated using the TD-DFT method as a function of the dissociation coordinate. The lowest excited state of alcohols was found to be repulsive, whereas a barrier to dissociation was found in the ethers. Rydbergvalence mixing and avoided crossings are decisive in determining the shapes of the potential surfaces. It is concluded that the reactivities of alcohols and ethers toward free electrons are rationalized by assuming that the potential surfaces of the daughter Feshbach resonances closely follow those of the parent Rydberg states, i.e., the lowest Feshbach resonance is repulsive, but a barrier occurs in ethers. The potential surfaces of both the Rydberg states and the Feshbach resonances thus differ dramatically from the non-dissociative surface of the grandparent ${ }^{2}\left(n^{-1}\right)$ positive ions, despite the nominally non-bonding character of the Rydberg electrons.

\section{Introduction}

Interest in chemical processes induced by free electrons is motivated by emerging applications in technology, for example focused electron beam induced processing (FEBIP), ${ }^{1}$ and the need to understand radiation-induced damage to living tissue. ${ }^{2}$ An important primary electron-induced process leading to chemical change is dissociative electron attachment (DEA), an example being the dissociation of methanol,

$$
\mathrm{e}^{-}\left(E_{\mathrm{i}}\right)+\mathrm{CH}_{3} \mathrm{OH} \rightarrow\left\{\mathrm{CH}_{3} \mathrm{OH}\right\}^{-} \rightarrow \mathrm{CH}_{3} \mathrm{O}^{-}+\mathrm{H}
$$

where $E_{\mathrm{i}}$ is the energy of the incident electron and the intermediate short-lived anion is called a resonance.

The present paper is concerned with DEA mediated by Feshbach resonances, situated generally between about 5 and $15 \mathrm{eV}$, where the incident electron causes a Rydberg excitation of the target molecules and is itself temporarily captured in a Rydberg-like orbital. Despite the somewhat exotic electronic structure of these core-excited resonances, they often represent the dominant path for DEA, particularly in saturated compounds. ${ }^{3,4}$ They appear to carry their signature even in the electron-induced damage to DNA in condensed phase. $^{2}$ Despite the very large body of experimental results on DEA, ${ }^{5,6}$ including many bands attributable to Feshbach resonances, virtually nothing is known about the detailed mechanism of the dissociation, in particular in polyatomic molecules. A notable exception is the recent calculation on the small molecule $\mathrm{H}_{2} \mathrm{O}{ }^{7,8}$

Department of Chemistry, University of Fribourg, chemin du Musée 9, Fribourg, CH-1700, Switzerland
In the present work we gain insight into the mechanism of dissociation of the Feshbach resonances in one particular case, that of alcohols and ethers. We first report a striking experimental observation, a difference of fragmentation patterns of the alcohols and the ethers, whereby the lowest Feshbach resonance ${ }^{2}\left(n, 3 \mathrm{~s}^{2}\right)$ yields strong $(M-1)$ signal in the alcohols, but no DEA signal at all in the ethers. We then note that this difference between the properties of the ${ }^{2}\left(n, 3 \mathrm{~s}^{2}\right)$ Feshbach resonances in the ethers and alcohols has an analogy in the spectroscopic properties of the parent ${ }^{1}(n, 3 \mathrm{~s})$ Rydberg states. Finally, we calculate the potential surfaces of the Rydberg states along the $\mathrm{O}-\mathrm{H}$ and $\mathrm{O}-\mathrm{C}$ bond stretching coordinate and show that they explain the Rydberg spectra and also the differences observed in the DEA spectra, under the assumption that the Feshbach resonance surfaces follow those of the parent Rydberg states.

\section{Methods}

\section{A Experimental}

The dissociative electron attachment spectrometer used to measure the yield of mass-selected stable anions as a function of electron energy has been described previously., ${ }^{4,10}$ It employs a magnetically collimated trochoidal electron monochromator $^{11}$ to prepare a beam of quasi-monoenergetic electrons, which is directed into a target chamber filled with a quasi-static sample gas. Fragment anions are extracted at $90^{\circ}$ by a three-cylinder lens and directed into a quadrupole mass spectrometer. The energy scale was calibrated on the onset of the $\mathrm{O}^{-} / \mathrm{CO}_{2}$ signal at $4.0 \mathrm{eV}$. The electron current was around $200 \mathrm{nA}$ and the resolution about $150 \mathrm{meV}$. Photoelectron 
spectra were recorded with a modified Perkin Elmer PS18 HeI photoelectron spectrometer. Electron energy loss spectrum (EELS) was recorded with a spectrometer using hemispherical electron-energy selectors. ${ }^{12}$

\section{B Calculations}

The excited state calculations were performed using the timedependent density functional theory (TD-DFT) PBE0/6$311++\mathrm{G}(3 \mathrm{df}, 3 \mathrm{p})$ model, the geometry optimizations using the DFT B3LYP/6-311 + G(2df,2p) model, as implemented in the Gaussian 03 package. ${ }^{13}$ This model has been tested and found to satisfactorily reproduce both valence and low-lying Rydberg states of a number of molecules. ${ }^{14}$ We confirmed this conclusion, since our calculated transition energies agree well with the experimental values. The calculated results for the $3 \mathrm{~s}$ and $3 \mathrm{p}_{x}$ states of methanol lie 0.12 and $0.36 \mathrm{eV}$ below our experimental values from the electron energy loss spectrum and a similar agreement is obtained for dimethyl and diethyl ethers when compared to the absorption spectra. ${ }^{15}$

Potential curves were obtained by calculating the transition energies over a range of $\mathrm{O}-\mathrm{H}$ and $\mathrm{O}-\mathrm{C}$ distances and adding them to the ground state energy calculated with the same model. Only the $\mathrm{O}-\mathrm{H}$ and $\mathrm{O}-\mathrm{C}$ distances were varied, the remaining geometrical parameters were not re-optimized (a rigid scan). The energy of the positive ion was calculated using the same model and used to plot the potential curve of the ground electronic state of the cation.

Rydberg states with large spatial extension can be poorly described by TD-DFT. ${ }^{16}$ One way to alleviate this problem ${ }^{16}$ is to increase the admixture of nonlocal Hartree-Fock (HF) exchange in hybrid functionals to values up to $50 \%$ as, e.g., in the BHLYP functional. ${ }^{16,17}$ We tested whether this problem effects our conclusions by repeating all calculations using the BHLYP functional, with the same basis set. The energies were calculated about $0.5 \mathrm{eV}$ higher than with the PBE0 model. Since the latter values were slightly lower than the experiment, BHLYP were somewhat higher, but satisfactorily close. The shapes of the potential energy curves were nearly identical with both methods.

Finally, we estimated the increase of the size of the electronic wave function upon excitation as the difference of the electronic spatial extents of a given excited state and the electronic ground state, $\Delta\left\langle r^{2}\right\rangle=\left\langle r^{2}\right\rangle-\left\langle r_{0}^{2}\right\rangle$. This calculation was done with the CIS model.

\section{Results and discussion}

Fig. 1 illustrates the well known ${ }^{20}$ relation between the grandparent cation, parent Rydberg state and daughter Feshbach resonance, on the example of the argon atom. ${ }^{21,22}$ Adding an electron into a Rydberg orbital around a positive ion core releases about $4 \mathrm{eV}$ of energy - the term value. Another about $0.4 \mathrm{eV}$, the electron affinity of the Rydberg state, is released when a second electron is added to an 3 s Rydberg state, leading to a short-lived Feshbach resonance involving two strongly correlated electrons localized on a potential-energy ridge. ${ }^{23}$ Both energies are to some degree independent of the molecule because of the weak penetration of the core by the diffuse Rydberg electron cloud, and this fact will be used to

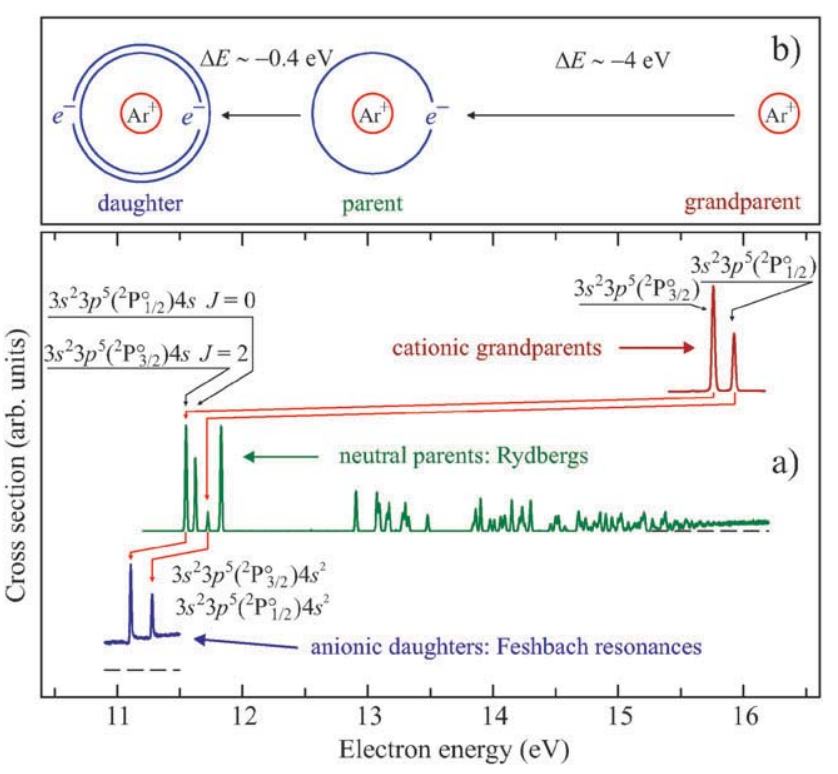

Fig. 1 Figure illustrating the descendancy relations between the grandparent cation, parent Rydberg and Feshbach anion states on the example of Ar. (a) The photoelectron spectrum (top), the electron energy loss spectrum (center), and the elastic cross section ${ }^{18}$ (bottom) of argon. (The energy-loss spectrum was recorded at a scattering angle of $\theta=135^{\circ}$ and a residual energy of $1 \mathrm{eV}$, the elastic cross section at $\theta=117^{\circ}$.) (b) Schematic diagram of the electron configurations, with the daughter Feshbach resonance having two highly correlated electrons moving in a Rydberg-like orbital.

predict the energies of the Feshbach resonances from ionization energies determined by He-I photoelectron spectra. The method is important in the present case of polyatomic molecules with Feshbach resonances without sharp structures, which can not be detected in elastic cross section or by other means.

A more detailed investigation of many molecules revealed that the $3 \mathrm{~s}$ term values are not entirely constant for different molecules, but decrease with increasing degree of alkylation, because bulky substituents penetrate even a large Rydberg orbital. ${ }^{15}$ As an example, the $3 \mathrm{~s}$ term value of ethanol is $3.8 \mathrm{eV}$, and this value decreases with increasing alkylation until it reaches a limiting value of about $2.8 \mathrm{eV}$ for very bulky alkyl substituents. ${ }^{15}$ Likewise, the energy relation between the Rydberg states and the Feshbach resonances has been studied in detail for a number of atoms and molecules, ${ }^{21,24-27}$ and is not entirely independent of the target.

Even with these limitations, the energy relation between the grandparent and the daughter represents a useful tool for assigning DEA bands to Feshbach resonances, as illustrated in Fig. 2. Ethanol and the two amines represent a series of compounds with gradually decreasing first ionization energy, and DEA bands closely following this trend are observed. They are situated below the 1st photoelectron band, by an amount of energy compatible with the above reasoning, permitting their assignment to the ${ }^{2}\left(n, 3 \mathrm{~s}^{2}\right)$ Feshbach resonances. The observations are not limited to the compounds shown in Fig. 2, but were made in all hydroxyl and amino group containing compounds which we studied. ${ }^{3,4}$ The relation permits even to assign the next DEA band to a Feshbach 


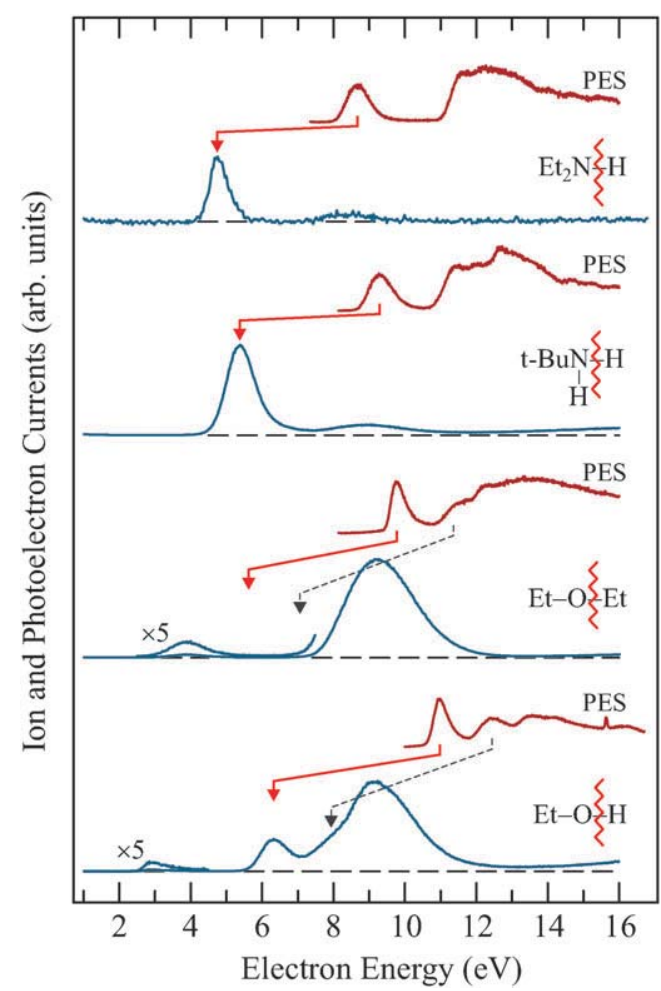

Fig. 2 Photoelectron spectra (PES) and DEA spectra of the compounds indicated. The bonds being broken are indicated in the formulas, the charge remains on the $\mathrm{O}$ or $\mathrm{N}$ containing fragment. Arrows indicate the relation between the grandparent ${ }^{2}\left(n^{-1}\right)$ positive ion states and the daughter ${ }^{2}\left(n, 3 \mathrm{~s}^{2}\right)$ Feshbach resonances, dashed arrows the ${ }^{2}\left(\bar{n}, 3 \mathrm{~s}^{2}\right)$ resonances. These two resonances apparently do not dissociate in diethylether, since no bands appear at the arrow positions. (The weak bands at $3 \mathrm{eV}$ in ethanol and $4 \mathrm{eV}$ in diethyl ether are due to shape resonances. ${ }^{4,19}$ )

resonance associated with the second ionization energy in alcohols, ${ }^{2}\left(\bar{n}, 3 \mathrm{~s}^{2}\right)$, as indicated for ethanol by the dashed arrow in Fig. 2. These resonances, belonging to deeper ionizations, were discussed in more detail earlier ${ }^{3,4}$ and are not of primary concern in this work.

The main experimental result of the present work is that no DEA bands corresponding to breaking a bond attached to the $\mathrm{O}$ atom appear around 5.2 and $7 \mathrm{eV}$ in diethyl ether, where Feshbach resonances are predicted by the analogy with the alcohols and the amines (the continuous and the dashed arrows above the Et-O-Et DEA spectrum in Fig. 2). There is little doubt that the ${ }^{2}\left(n, 3 \mathrm{~s}^{2}\right)$ and the ${ }^{2}\left(\bar{n}, 3 \mathrm{~s}^{2}\right)$ Feshbach resonances, with holes in the oxygen lone pair orbitals, occur at these energies.

It is unlikely that the absence of DEA bands from these resonances is due to a very large autodetachment width $\Gamma$ and, thus, an unfavorable competition between dissociation and autodetachment in the ether. The autodetachment widths of Feshbach resonances are generally narrow, substantially narrower than a typical vibrational spacing, both in atoms (Fig. 1) and in molecules (for example in acetylene ${ }^{28}$ or acetaldehyde ${ }^{29}$ ). The large width of the spectral bands in the present molecules must be due to wide Franck-Condon profiles with many overlapping vibrations or to a repulsive potential surface. We thus expect the Feshbach resonances to have a narrow $\Gamma$ in both alcohols and ethers.

In this case the absence of ${ }^{2}\left(n, 3 \mathrm{~s}^{2}\right)$ DEA band in the ether must indicate that this resonance is, in contrast to the corresponding resonances in the alcohols and amines, not dissociative. This conclusion is not limited to diethyl ether shown in Fig. 2, but applies to all ethers which we have studied so far, including dibutyl ether. ${ }^{19}$

In search for the explanation of this experimental result we note that a related observation has been made for the parent Rydberg states - it is illustrated for the case of methanol in Fig. 3 (see also ref. 30). Robin analyzed term energies and band shapes in VUV spectra and recognized that in water and the alcohols the lowest ${ }^{1}(n, 3 \mathrm{~s})$ Rydberg states are anomalous in the sense that they are broad and structureless-whereas they may be expected to be narrow and have a sharp vibrational structure like the corresponding photoelectron bands (this expectation is fulfilled for the higher-lying ${ }^{1}(n, 3 \mathrm{p})$ bands in Fig. 3). ${ }^{15}$ In contrast, the lowest Rydberg bands in the ethers behave normally - they have a sharp vibrational structure, similar to that of the grandparent ion in the photoelectron spectrum. Robin concluded that the ${ }^{1}(n, 3 \mathrm{~s})$ Rydberg states are strongly perturbed by the conjugate ${ }^{1}\left(n, \sigma^{*}\right)$ valence promotions in the alcohols and water, but that this perturbation is not significant in the ethers. It is interesting to note that G. Herzberg already realized in 1931 that in the model compound $\mathrm{H}_{2} \mathrm{O}$ the absence of structure indicates that the $3 \mathrm{~s}$ state is repulsive, in contrary to an initial expectation for a Rydberg state (see the comment by G. Herzberg in the general discussion at the end of ref. 31).

We gain further insight into the causes for this behavior of the Rydberg states by calculating the potential energy curves along the dissociation coordinates. To simplify the calculation and the orbital diagrams, we present the curves for the smaller molecules methanol and methyl ethyl ether instead of ethanol and diethyl ether shown in Fig. 2-this does not affect the argument since the observations appear to apply to all alcohols and ethers. The results are shown in Fig. 4 and 5.

The potential energy curves are compatible with the energyloss spectrum of Fig. 3 in the sense that the structureless $3 \mathrm{~s}$

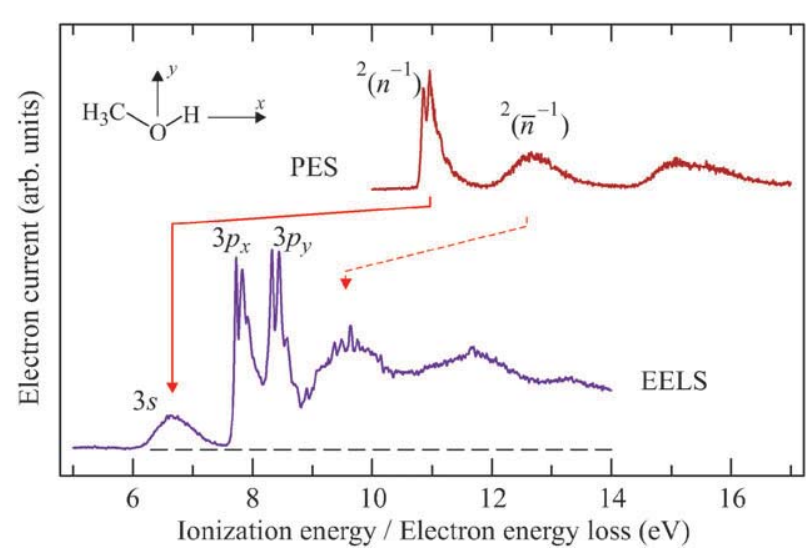

Fig. 3 The photoelectron (top) and the electron energy loss spectrum of methanol (the latter recorded at a scattering angle of $0^{\circ}$ and a residual energy of $10 \mathrm{eV}$ ). 


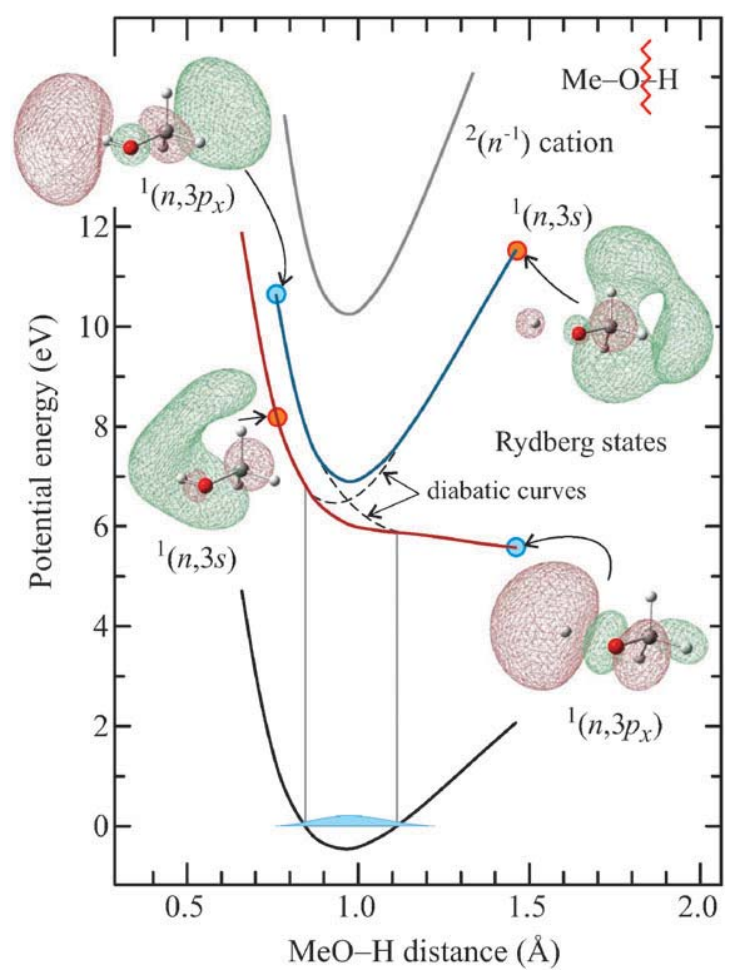

Fig. 4 Potential energy surface of methanol (bottom curve), its two lowest excited states, and the ground state of the cation (top curve), shown as a function of the $\mathrm{O}-\mathrm{H}$ distance.

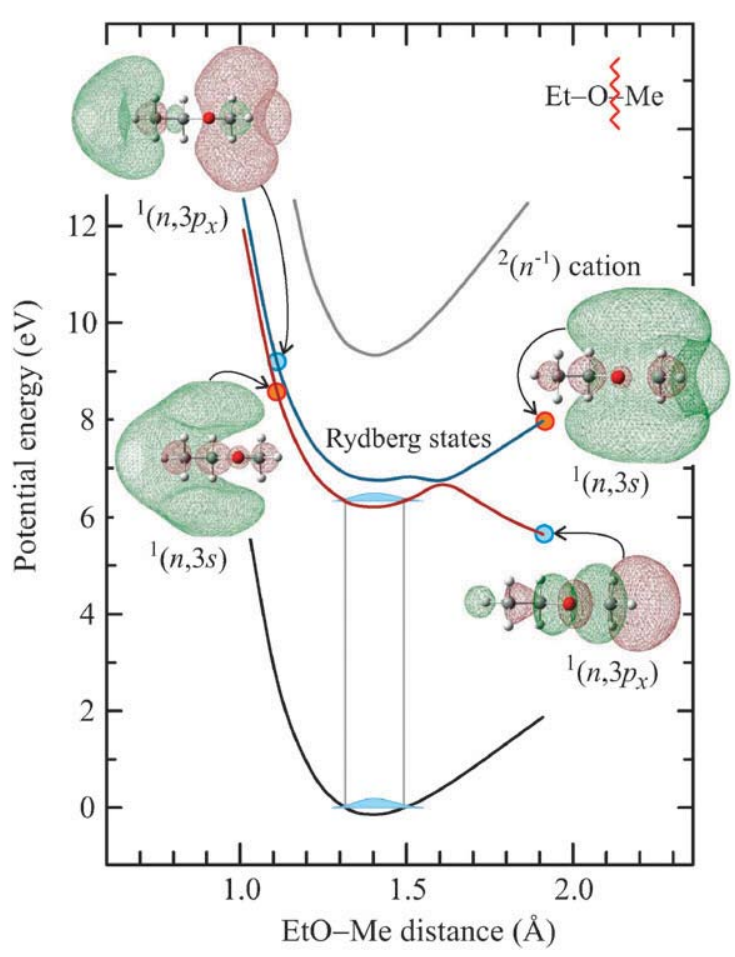

Fig. 5 Same as Fig. 4, but for methyl ethyl ether.

band indicates a dissociative potential whereas the structured $3 \mathrm{p}_{x}$ band indicates that the $3 \mathrm{p}_{x}$ potential is not dissociative. Calculation of the next excited states, $3 \mathrm{p}_{y}$ and $3 \mathrm{p}_{z}$, yields potential curves with shapes similar to that of the positive ion (shown at the top of Fig. 4). The $3 p_{y}$ and $3 p_{z}$ curves are not shown in the Figure to improve clarity. The calculated $3 \mathrm{p}_{y}$ state explains well the $3 \mathrm{p}_{y}$-labeled band in Fig. 3 . The $3 \mathrm{p}_{z}$ state has an energy only slightly higher than that of the $3 \mathrm{p}_{y}$ state and a very low oscillator strength, and is consequently not easily visible in the energy-loss spectrum.

The important difference between the curves of the alcohol in Fig. 4 and the ether in Fig. 5 is that in the latter there is a barrier to dissociation on the lower curve. This explains why the 3s VUV absorption band of ethers does have, in contrast to the alcohols, vibrational structure. ${ }^{15}$

The behavior of these potential energy surfaces follows the same fundamental pattern studied in detail for the ${ }^{1}\left(\pi, \sigma^{*}\right)$ states of a number of molecules of biological relevance, ${ }^{32,33}$ with the difference that the present molecules do not have a $\pi, \pi^{*}$ chromophore. These studies revealed that the ${ }^{1}\left(\pi, \sigma^{*}\right)$ state can be classified as a 3s Rydberg state that collapses in terms of size upon the stretching of the $\mathrm{OH}$ bond (for example in phenol) or the $\mathrm{NH}$ bond (for example in pyrrole and indole). This Rydberg-to-valence orbital transformation was reflected by a double-well shape of the ${ }^{1}\left(\pi, \sigma^{*}\right)$ potential energy function in some molecules. ${ }^{32,33} \mathrm{~A}$ diffuse ${ }^{1}\left(\pi, \sigma^{*}\right)$ state was also invoked in the photodissociation of the $\mathrm{C}-\mathrm{O}$ bond in aromatic compounds. ${ }^{34}$ Related $\pi^{*}-\sigma^{*}$ potential energy surfaces were invoked by Barrios et al. ${ }^{35}$ to interpret the dissociation of a portion of DNA following an attachment of an electron, albeit via a shape resonance.

The present curves, particularly for the ether (Fig. 5) are also remarkably similar to those of the ${ }^{3} \Pi_{u}$ manifold of $\mathrm{O}_{2}{ }^{36}$ The shapes of the $\mathrm{O}_{2}$ curves were explained as a consequence of predissociation of the nominally bound Rydberg curves by a strongly repulsive valence curve, that is, a consequence of avoided crossings resulting from Rydberg-valence coupling. This suggests that also in the present case the repulsive nature of the nominally $3 \mathrm{~s}$ states, the barrier to dissociation in the ether, and the double minimum on the upper state in the ether, can all be understood as a result of avoided crossings.

The excited state orbitals are informative about the nature of the excited state and are shown in Fig. 4 and 5 for two O-H and $\mathrm{O}-\mathrm{C}$ bond distances, one shorter and one longer than the equilibrium bond length. For both compounds the orbitals of the lower states on the left, at short bond distances, are reminiscent of $3 \mathrm{~s}$ orbitals. The spherical symmetry is perturbed by the non-spherical core and the outer layer does not entirely enclose the core (the degree to which it encloses it depends on the iso value chosen for the rendition), but the tendency of the outer cloud to enclose the core is unmistakable. The orbitals of the second excited states on the left are clearly $3 p_{x}$-like (see Fig. 3 for the orientation of the coordinates). This ordering corresponds to the spectroscopic assignment of the Rydberg bands in the UV and energy-loss spectra and is also compatible with the values of the quantum defects derived from the experimental energies. ${ }^{15}$ They are $\delta=1.2$ and $\delta=0.84$ for the 3 s states, and $\delta=0.95$ and $\delta=0.56$ for the $3 \mathrm{p}_{x}$ states, for methanol and diethyl ether, respectively.

The Rydberg nature of the states was further confirmed by the calculated (see section IIB) spatial extent of the electronic wave function, $\Delta\left\langle r^{2}\right\rangle$, which was $17 a_{0}^{2}$ for the 3 s and $33 a_{0}^{2}$ for the $3 p_{x}$ state in methanol. For the lower state, $\Delta\left\langle r^{2}\right\rangle$ decreased 
to about $7 a_{0}^{2}$ as the $\mathrm{O}-\mathrm{H}$ bond was stretched, reflecting a Rydberg-valence conversion. In contrast, for the upper, nominally $3 \mathrm{p}_{x}$ state, $\Delta\left\langle r^{2}\right\rangle$ remained constant at a value characteristic for a Rydberg state, around $30 a_{0}^{2}$. For methyl ethyl ether the $\Delta\left\langle r^{2}\right\rangle$ were $30 a_{0}^{2}$ for the $3 \mathrm{~s}$ and $41 a_{0}^{2}$ for the $3 \mathrm{p}_{x}$ state. As for the alcohol, the spatial extent of the $3 \mathrm{~s}$ state decreased to about $10 a_{0}^{2}$ when the $\mathrm{O}-\mathrm{Me}$ bond was stretched onto the repulsive section of the potential, beyond the activation barrier (Fig. 5), whereas that of the $3 p_{x}$ state remained above $30 a_{0}^{2}$.

The nature of the orbitals at large $\mathrm{O}-\mathrm{H}$ bond lengths, on the right in Fig. 3, appears reversed, the upper orbital resembles more $3 \mathrm{~s}$ and the lower $3 \mathrm{p}_{x}$ (or $\left.\sigma^{*}\right)$. This could be understood as an indication an avoided crossing of the $3 \mathrm{~s}$ and $3 \mathrm{p}_{x}$ states (both have the $A^{\prime \prime}$ symmetry), as tentatively indicated in the Figure. Note that the $3 \mathrm{p}_{x}$ orbital in methanol has a pronounced node across the $\mathrm{O}-\mathrm{H}$ bond (Fig. 4) - for this low principal quantum number the nominally Rydberg orbital has a partial $\sigma^{*}$ character.

The details of the potential curve of the 2 nd excited state of the ether (Fig. 5) are more complicated than in methanol. The $3 \mathrm{p}_{x}$ orbital in the ether appears less antibonding across the $\mathrm{O}-\mathrm{C}$ bond than was the case in methanol across the $\mathrm{O}-\mathrm{H}$ bond. The potential curve of the 2 nd excited state has a shallow double minimum indicative of another avoided crossing with a strongly repulsive $\sigma^{*}$ state. (This repulsive state can be followed to higher energies, where avoided crossings with higher-lying $A^{\prime \prime}$ Rydberg states-not shown in Fig. 5appear.) These details of the potential, however, do not affect our main conclusion that the 1st excited state is dissociative in the alcohols, but bound in the ethers.

As a final step of our reasoning we note that the main experimental observation of this paper, namely the fact that the ${ }^{2}\left(n, 3 \mathrm{~s}^{2}\right)$ Feshbach resonance gives rise to a strong DEA signal in the alcohols, but no detectable DEA signal in the ethers, can be explained by assuming that the main features of the Rydberg potential curves shown in Fig. 4 and 5 apply also to the Feshbach resonances. Since the additional electron in a Feshbach resonance is in a diffuse orbital and is only weakly bound, it is not unreasonable to assume that it does not substantially contribute to bonding and consequently does not appreciably change the shape of the potential curves, except for lowering them by about $0.4 \mathrm{eV}$.

We also calculated the potential curves for the higher-lying Rydberg states, the $A^{\prime}{ }^{1}(\bar{n}, 3 \mathrm{~s})$ and ${ }^{1}\left(\bar{n}, 3 \mathrm{p}_{x}\right)$ where $\bar{n}$ is the $2 p_{y^{-}}$ like $a^{\prime}$ lone pair orbital on the $\mathrm{O}$ atom. The results were similar in terms of the shapes to the curves discussed above, the nominally $3 \mathrm{~s}$ states being dissociative in the alcohol and not dissociative in the ether. These results are in line with the observation of a DEA band assigned as ${ }^{2}\left(\bar{n}, 3 \mathrm{~s}^{2}\right)$ in the alcohols and its absence in the ethers (see Fig. 2).

\section{Conclusions}

The present work reveals a qualitative difference in the dissociation patterns of Feshbach resonances in alcohols and in ethers. Whereas the $\mathrm{O}-\mathrm{H}$ bond cleavage in alcohols already proceeds from the lowest Feshbach resonance (with a hole in the oxygen lone pair orbital), the $\mathrm{C}-\mathrm{O}$ bond cleavage in ethers proceeds only from the higher-lying Feshbach resonances with holes in the $\sigma_{\mathrm{C}-\mathrm{C}}$ and $\sigma_{\mathrm{C}-\mathrm{H}}$ orbitals. From this observation we conclude that the lowest Feshbach resonance, ${ }^{2}\left(n, 3 \mathrm{~s}^{2}\right)$, is dissociative in the alcohols, but bound in the ethers.

We further point out the spectral evidence that the same behavior is found for the parent states of these Feshbach resonances, the ${ }^{1}(n, 3 \mathrm{~s})$ Rydberg states.

We then calculated the potential curves for the Rydberg states along the dissociation coordinate and found that they explain the observed spectral properties, a broad structureless band in the alcohols, and a band with vibrational structure in the ethers. Finally we conclude that, under the assumption that an additional electron in a diffuse orbital does not appreciably change the shape of the potential curves, these potential curves also explain the observations for DEA to these molecules.

These calculations provide an insight into a situation which is a priori surprising. A simple view of the Rydberg electron is that it is in a spatially large, diffuse cloud, does not penetrate the positive ion core, and does not contribute to chemical binding. This view would result in an expectation that the potential surfaces of the ${ }^{2}\left(n, 3 \mathrm{~s}^{2}\right)$ Feshbach resonances follow those of the ${ }^{2}\left(n^{-1}\right)$ positive ion core, which are not dissociative. No DEA signal would thus be expected from the ${ }^{2}\left(n, 3 \mathrm{~s}^{2}\right)$ Feshbach resonances. Our study suggests that a $\sigma^{*}$ orbital, which has, in terms of its nodal structure, partially a $3 \mathrm{p}_{x}$ character, is responsible for the dissociative behavior. An avoided crossing has then the consequence that it is the nominally ${ }^{2}\left(n, 3 \mathrm{~s}^{2}\right)$ Feshbach resonance which is predissociated by the repulsive state, explaining the DEA signal. The difference between the alcohols and the ethers is the absence or presence of an energy barrier on the lower state potential energy surface.

Although not discussed in detail in this work, similar behavior was found also for higher excited states and Feshbach resonances, with occupation of the same $3 \mathrm{~s}, 3 \mathrm{p}_{x}$ and $\sigma^{*}$ orbitals, but a hole in the next deeper lying orbital $\bar{n}$ (essentially a $2 p_{y}$ orbital, with a considerable $\sigma_{\mathrm{C}-\mathrm{O}}$ character).

The principal finding of this work, the dissociative nature of Feshbach resonance centered on a hydroxyl oxygen and nondissociative nature of Feshbach resonance centered on a ether oxygen, is preserved even in compounds having hydroxyl and ether oxygens in one molecule. Based on photoelectron spectra, two distinct Feshbach resonances, ${ }^{2}\left(n_{\text {ether }}, 3 \mathrm{~s}^{2}\right)$ at lower energy and ${ }^{2}\left(n_{\mathrm{OH}}, 3 \mathrm{~s}^{2}\right)$ at higher energy, were identified in tetrahydrofuran-3-ol and (tetrahydrofuran-2-yl)methanol, but only the hydroxyl-centered ${ }^{2}\left(n_{\mathrm{OH}}, 3 \mathrm{~s}^{2}\right)$ Feshbach resonance resulted in DEA signal. ${ }^{4}$

As an outlook, it would be interesting to calculate directly the potential curves of the Feshbach resonances using a scattering calculation such as the R-matrix theory yielding both their energy and the autodetachment width.

\section{Acknowledgements}

We thank to Olivier May for assistance in recording the photoelectron spectra and acknowledge helpful discussions with W. Domcke, H. Hotop and S. Grimme. This research 
is part of project No. 200020-113599/1 of the Swiss National Science Foundation.

\section{References}

1 I. Utke, V. Friedli, M. Purrucker and J. Michler, J. Vac. Sci. Technol., B, 2007, 25(6), 2219.

2 B. Boudaiffa, P. Cloutier, D. Hunting, M. A. Huels and L. Sanche, Science, 2000, 287, 1658.

3 T. Skalický and M. Allan, J. Phys. B, 2004, 37, 4849.

4 B. C. Ibănescu, O. May, A. Monney and M. Allan, Phys. Chem. Chem. Phys., 2007, 9, 3163.

5 L. G. Christophorou, D. L. McCorkle and A. A. Christodoulides, in Electron-Molecule Interactions and their Applications, ed. L. G. Christophorou, Academic Press, Orlando, 1984, vol. 1, p. 477.

6 E. Illenberger and J. Momigny, Gaseous Molecular Ions, Steinkopff Verlag, Darmstadt and Springer Verlag, New York, 1992.

7 D. J. Haxton, T. N. Rescigno and C. W. McCurdy, Phys. Rev. A, 2007, 75, 012711

8 D. J. Haxton, T. N. Rescigno and C. W. McCurdy, Phys. Rev. A, 2007, 76, 049907 (erratum).

9 M. Stepanović, Y. Pariat and M. Allan, J. Chem. Phys., 1999, 110, 11376.

10 R. Dressler and M. Allan, Chem. Phys., 1985, 92, 449.

11 A. Stamatovic and G. J. Schulz, Rev. Sci. Instrum., 1968, 39 1752.

12 M. Allan, J. Phys. B, 1992, 25, 1559.

13 M. J. Frisch, G. W. Trucks, H. B. Schlegel, G. E. Scuseria, M. A. Robb, J. R. Cheeseman, J. A. Montgomery, Jr., T. Vreven, K. N. Kudin, J. C. Burant, J. M. Millam, S. S. Iyengar, J. Tomasi, V. Barone, B. Mennucci, M. Cossi, G. Scalmani, N. Rega, G. A. Petersson, H. Nakatsuji, M. Hada, M. Ehara, K. Toyota, R. Fukuda, J. Hasegawa, M. Ishida, T. Nakajima, Y. Honda, O. Kitao, H. Nakai, M. Klene, X. Li, J. E. Knox, H. P. Hratchian, J. B. Cross, V. Bakken, C. Adamo, J. Jaramillo, R. Gomperts, R. E. Stratmann, O. Yazyev, A. J. Austin, R. Cammi, C. Pomelli, J. Ochterski, P. Y. Ayala, K. Morokuma, G. A. Voth, P. Salvador, J. J. Dannenberg, V. G. Zakrzewski, S. Dapprich, A. D. Daniels, M. C. Strain, O. Farkas, D. K. Malick, A. D. Rabuck, K.
Raghavachari, J. B. Foresman, J. V. Ortiz, Q. Cui, A. G. Baboul, S. Clifford, J. Cioslowski, B. B. Stefanov, G. Liu, A. Liashenko, P. Piskorz, I. Komaromi, R. L. Martin, D. J. Fox, T. Keith, M. A. Al-Laham, C. Y. Peng, A. Nanayakkara, M. Challacombe, P. M. W. Gill, B. G. Johnson, W. Chen, M. W. Wong, C. Gonzalez and J. A. Pople, GAUSSIAN 03 (Revision C.01), Gaussian, Inc., Wallingford, CT, 2004.

14 I. Cioffini and C. Adamo, J. Chem. Phys., 2007, 111, 5549.

15 M. B. Robin, Higher Excited States of Polyatomic Molecules, Academic Press, New York, vol. 1, 1974.

16 S. Grimme and F. Neeseb, J. Chem. Phys., 2007, 127 154116.

17 A. D. Becke, J. Chem. Phys., 1993, 98, 1372.

18 K. Franz, T. H. Hoffmann, J. Bömmels, A. Gopalan, G. Sauter, W. Meyer, M. Allan, M.-W. Ruf and H. Hotop, Phys. Rev. A, 2008 , in preparation.

19 B. C. Ibănescu, O. May and M. Allan, Phys. Chem. Chem. Phys., 2008, 10, 1507.

20 G. J. Schulz, Rev. Mod. Phys., 1973, 45, 423.

21 L. Sanche and G. J. Schulz, Phys. Rev. A, 1972, 5, 1672.

22 D. Spence, Phys. Rev. A, 1977, 15, 883.

23 U. Fano and A. R. P. Rau, Comments At. Mol. Phys., 1985, 16, 241.

24 L. Sanche and G. J. Schulz, Phys. Rev. A, 1972, 6, 69.

25 L. Sanche and G. J. Schulz, J. Chem. Phys., 1973, 58, 479.

26 D. Spence, J. Phys. B, 1975, 12, 721.

27 D. Spence, J. Chem. Phys., 1977, 66, 669.

28 R. Dressler and M. Allan, J. Chem. Phys., 1987, 87, 4510.

29 R. Dressler and M. Allan, J. Electron Spectrosc. Relat. Phenom., 1986, 41, 275.

30 M. B. Robin and N. A. Kuebler, J. Electron Spectrosc. Relat. Phenom., 1972, 1, 13.

31 C. F. Goodeve and N. O. Stein, Trans. Faraday Soc., 1931, 21, 393.

32 A. L. Sobolewski, W. Domcke, C. Dedonder-Lardeux and C. Jouvet, Phys. Chem. Chem. Phys., 2002, 4, 1093.

33 A. L. Sobolewski and W. Domcke, J. Phys. Chem. A, 2007, 111, 11725 .

34 S. Grimme, Chem. Phys., 1992, 163, 313.

35 R. Barrios, P. Skurski and J. Simons, J. Phys. Chem. B, 106(33), 7991.

36 B. R. Lewis, J. P. England, S. T. Gibson, M. J. Brunger and M. Allan, Phys. Rev. A, 2001, 63, 022707. 